\title{
Scaling of the Low-Temperature Dephasing Rate in Kondo Systems
}

\author{
F. Mallet, ${ }^{1,2}$ J. Ericsson, ${ }^{1,2}$ D. Mailly, ${ }^{4}$ S. Ünlübayir, ${ }^{5}$ D. Reuter, ${ }^{5}$ A. Melnikov, ${ }^{5}$ A. D. Wieck, ${ }^{5}$ T. Micklitz, ${ }^{6}$ A. Rosch, ${ }^{6}$ \\ T. A. Costi, ${ }^{7}$ L. Saminadayar, ${ }^{1,2,3}$ and C. Bäuerle ${ }^{1,2}$ \\ ${ }^{1}$ Centre de Recherches sur les Très Basses Températures, B.P. 166 X, 38042 Grenoble Cedex 09, France \\ ${ }^{2}$ Institut Néel, B.P. 166 X, 38042 Grenoble Cedex 09, France \\ ${ }^{3}$ Université Joseph Fourier, B.P. 53, 38041 Grenoble Cedex 09, France \\ ${ }^{4}$ Laboratoire de Photonique et Nanostructures, route de Nozay, 91460 Marcoussis, France \\ ${ }^{5}$ Lehrstuhl für Angewandte Festkörperphysik, Ruhr-Universität, Universitätsstr. 150, 44780 Bochum, Germany \\ ${ }^{6}$ Institute for Theoretical Physics, University of Cologne, 50937 Cologne, Germany \\ ${ }^{7}$ Institut für Festkörperforschung, Forschungszentrum Jülich, 52425 Jülich, Germany
}

(Received 6 July 2006; published 30 November 2006)

\begin{abstract}
We present phase coherence time measurements in quasi-one-dimensional Ag wires doped with $\mathrm{Fe}$ Kondo impurities of different concentrations $n_{s}$. Because of the relatively high Kondo temperature $T_{K} \approx$ $4.3 \mathrm{~K}$ of this system, we are able to explore a temperature range from above $T_{K}$ down to below $0.01 T_{K}$. We show that the magnetic contribution to the dephasing rate $\gamma_{m}$ per impurity is described by a single, universal curve when plotted as a function of $T / T_{K}$. For $T>0.1 T_{K}$, the dephasing rate is remarkably well described by recent numerical results for spin $S=1 / 2$ impurities. At lower temperature, we observe deviations from this theory. Based on a comparison with theoretical calculations for $S>1 / 2$, we discuss possible explanations for the observed deviations.
\end{abstract}

DOI: 10.1103/PhysRevLett.97.226804

PACS numbers: 73.23.-b, 72.15.Qm, 73.20.Fz, 75.20.Hr

The Kondo effect has been a central theme in solid state physics for several decades. The fascination it still arouses is due to the fact that it represents a paradigm of the generic problem of dynamical impurities in metals [1]. Its most well-known manifestation is the logarithmic increase of the low-temperature resistivity of metals containing magnetic impurities [2]. The phenomenon is accompanied by a progressive screening of the spin of the impurities by the surrounding conduction electrons, resulting, in the case of complete screening, in a Fermi liquid ground state [3]. In the last decade, a number of experiments have also demonstrated [4-7] that Kondo impurities provide an efficient mechanism for electronic decoherence at low temperature. Conversely, measurement of the phase coherence time is a powerful tool to probe the ground state of a Kondo system [8].

On the theoretical side, it has been shown very recently [9] that the dephasing rate of electrons scattering from diluted Kondo impurities can be calculated exactly from the inelastic scattering cross section [10] by using Numerical Renormalization Group (NRG) techniques. The calculated full temperature dependence of the dephasing rate [9] thereby allows for a quantitative comparison between experimental data and theoretical predictions, bridging the gap between the low-temperature Fermi liquid theory and the "high-"temperature Nagaoka-Suhl expansion. Indeed, recent experiments [11] have confirmed qualitative aspects of these NRG calculations; in particular, the dephasing rate has been found to be linear with temperature over almost one decade below $T_{K}$. The relatively low Kondo temperature of the system used in that work, on the other hand, did not allow to perform measurements well below $0.1 T_{K}$ where the Fermi liquid regime should appear. In this context, a natural challenge is to investigate the very low-temperature limit of the magnetic contribution to the dephasing rate $\gamma_{m}$.

In this Letter, we present measurements of the phase coherence time $\tau_{\phi}$ of $\mathrm{AgFe}$ Kondo wires with different magnetic impurity concentrations and down to temperatures of $0.01 T_{K}$. We show that the magnetic contribution to the dephasing rate $\gamma_{m}$ per magnetic impurity presents a universal scaling when plotted as a function of $T / T_{K}$. This dephasing rate is remarkably well described by the recent numerical result for spin $S=1 / 2$ impurities $[9,10]$. On the other hand, at very low temperatures $\left(T<0.1 T_{K}\right)$, we observe deviations from this result.

In order to be able to explore the very low-temperature limit of Kondo systems, several severe experimental requirements have to be met. First of all, a suitable Kondo system with a relatively high Kondo temperature is needed in order to be able to attain temperatures of the order of $0.01 T_{K}$. At the same time, one would like to avoid that the Kondo maximum of the dephasing rate is masked by the phonon contribution. Secondly, one has to be able to start with a very pure sample which already shows good agreement with theory in the absence of magnetic impurities. In addition, one would like to be able to use identical samples and add magnetic impurities with a very high precision.

A Kondo system which satisfies the above conditions is $\mathrm{Ag} / \mathrm{Fe}$ with a Kondo temperature of around $3 \mathrm{~K}$ [12]. For this system, we can easily attain temperatures well below $0.1 T_{K}$. In addition, we are able to prepare extremely pure silver samples [4]. The idea is hence to fabricate several identical quantum wires (see inset of Fig. 1) on the same 
TABLE I. Sample characteristics: $l, R, \rho$, and $D$ correspond to the length, electrical resistance, resistivity, and diffusion coefficient. $n_{s}$ corresponds to the nominal impurity concentration measured via the ion current during the implantation. $n_{s}^{\gamma}$ is the impurity concentration extracted from fitting $\gamma_{m}$ to the NRG $S=1 / 2$ theory. All samples have a width of $w=150 \mathrm{~nm}$ and thickness of $t=50 \mathrm{~nm}$.

\begin{tabular}{lcccccc}
\hline \hline Sample & $\begin{array}{c}l \\
(\mu \mathrm{m})\end{array}$ & $\begin{array}{c}R \\
(\Omega)\end{array}$ & $\begin{array}{c}\rho \\
(\mu \Omega \mathrm{cm})\end{array}$ & $D$ & $\begin{array}{c}n_{s} \\
(\mathrm{ppm})\end{array}$ & $\begin{array}{c}n_{s}^{\gamma} \\
(\mathrm{ppm})\end{array}$ \\
\hline AgFe1 & 745 & 877 & 0.88 & 429 & 2.7 & 1.3 \\
$\mathrm{AgFe} 2$ & 405 & 517 & 0.96 & 400 & 27 & 13 \\
$\mathrm{AgFe} 3$ & 160 & 222 & 1.04 & 360 & 67.5 & 33 \\
$\mathrm{Ag} 2$ & 765 & 1305 & 1.28 & 295 & 0 & $\cdots$ \\
\hline \hline
\end{tabular}

wafer. Subsequently, the samples are implanted in a very controlled manner with magnetic impurities via Focused Ion Beam (FIB) technology. The $\mathrm{Fe}^{+}$ion implantation is done with an energy of $100 \mathrm{keV}$ and the implanted ion concentration $n_{s}$ is determined directly via the ion current [13]. In Table I, we summarize the parameters of our samples.

We define $T_{K}^{\rho}$ as the Kondo temperature extracted from resistivity measurements. The electrical resistance of a quasi one-dimensional metallic wire containing magnetic impurities at low temperature is given by

$$
R(T)=R_{0}+\alpha / \sqrt{T}+n_{s} * f\left(T / T_{K}^{\rho}\right)
$$

where $R_{0}$ is the residual resistance and $n_{s}$ is the magnetic impurity concentration. The second term corresponds to the electron-electron interaction term $[14,15]$ and the third term to the Kondo contribution. The electron-electron interaction contribution can be determined independently by measuring the resistivity of a clean sample ( $\mathrm{Ag} \mathrm{2}$ ) containing no magnetic impurities. Fitting the temperature dependence of the resistance variation to $\Delta R(T)=$ $\alpha_{\text {exp }} / \sqrt{T}$, we obtain $\alpha_{\text {exp }}=0.081 \Omega \mathrm{K}^{1 / 2}$, in good agreement with the theoretical value $\alpha_{\text {theo }}=2 R^{2} / R_{K} /$ $L \sqrt{\hbar D / k_{B}}=0.082 \Omega \mathrm{K}^{1 / 2}$, where $R_{K}=h / e^{2}$ is the quantum of resistance. The Kondo contribution to the resistivity per impurity concentration is shown in Fig. 1 for sample $\mathrm{AgFe} 2$ and $\mathrm{AgFe} 3$. Both data sets scale nicely with impurity concentration, and one observes typical features of a Kondo system: a logarithmic increase below the Kondo minimum and a saturation at the lowest temperatures. Fitting the resistivity data to the NRG results for $S=1 / 2[16,17]$, we obtain a Kondo temperature of $T_{K}^{\rho}=3.0 \pm 0.3 \mathrm{~K}$.

Phase coherence measurements, on the other hand, are much more sensitive to the presence of magnetic impurities. Here we determine the phase coherence time $\tau_{\phi}$ as displayed in Fig. 2 by fitting the low field magnetoresistance to standard weak localization theory $[14,18]$.

The experimentally measured phase coherence time $\tau_{\phi}$ for a metallic quantum wire containing magnetic impuri-

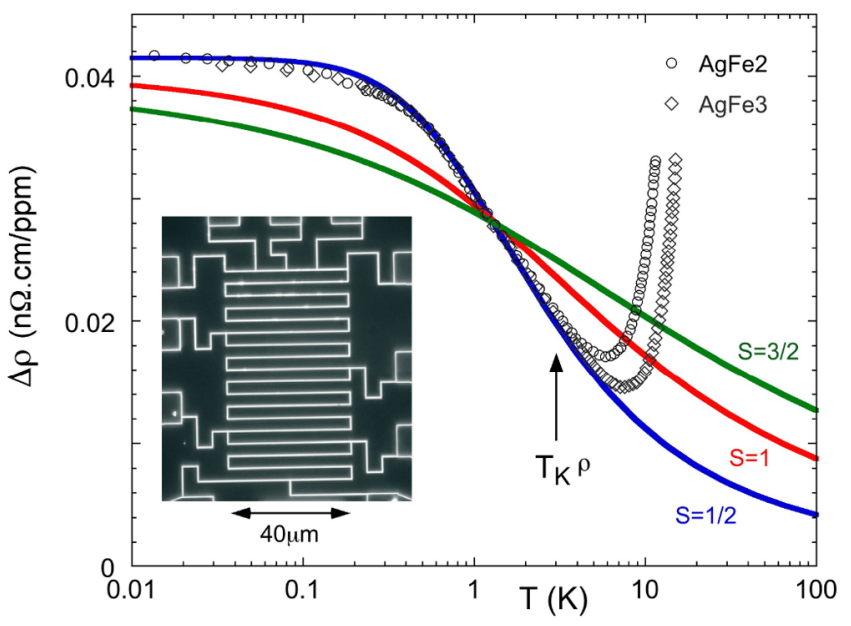

FIG. 1 (color online). Resistivity per magnetic impurity concentration as a function of temperature for sample $\mathrm{AgFe} 2$ and AgFe3. The experimental data have been superposed at low temperatures and the electron-electron contribution has been subtracted. The solid curved lines are the NRG results for $S=1 / 2, S=1$, and $S=3 / 2$.

ties can be described by the following expression [19]

$$
\frac{1}{\tau_{\phi}}=\frac{1}{\tau_{e-e}}+\frac{1}{\tau_{e-\mathrm{ph}}}+\frac{1}{\tau_{m}}
$$

where

$$
\frac{1}{\tau_{e-e}}=a_{\text {theo }} T^{2 / 3}=\left[\frac{\pi}{\sqrt{2}} \frac{R}{R_{K}} \frac{k_{B}}{\hbar} \frac{\sqrt{D}}{L}\right]^{2 / 3} T^{2 / 3}
$$

corresponds to the electron-electron interaction term [15], $1 / \tau_{e-\mathrm{ph}}=b T^{3}$ to the electron-phonon interaction, while $1 / \tau_{m}=\gamma_{m}$ corresponds to the magnetic contribution to the dephasing. In absence of magnetic scattering, the temperature dependence of the phase coherence time is determined by the first two terms in Eq. (2) [14]. Our data on the clean wire (sample $\mathrm{Ag} 2$ ) follow nicely the expected temperature dependence down to $40 \mathrm{mK}$ (see Fig. 2). From fitting the data, we extract $a_{\text {exp }}=0.42 \mathrm{~ns}^{-1} \mathrm{~K}^{-2 / 3}$ and $b=$ $0.04 \mathrm{~ns}^{-1} \mathrm{~K}^{-3}$ [22]. At very high temperatures $(T>5 K)$, all samples follow essentially the same temperature dependence. In this regime, the phase coherence time is mainly determined by electron-phonon scattering.

The phase coherence time $\tau_{\phi}$ for samples containing magnetic Fe impurities shows a quite different behavior. One first observes a plateau around $2 \mathrm{~K}$ and subsequently a desaturation of the phase coherence time. This can be understood within the framework of Kondo physics. At temperatures above the Kondo scale $T_{K}$ spin flip scattering is dominant and leads to a plateau in $\tau_{\phi}$. At temperatures below $T_{K}$, the magnetic impurity spins get screened and the phase coherence time increases. Using the NRG results for $S=1 / 2$ [9] to determine the magnetic contribution to the dephasing in Eq. (2), we can fit the experimental data (see Fig. 2) and extract $T_{K}^{\gamma}$ as well as the magnetic impurity concentration $n_{s}^{\gamma}$ using the value $\nu=1.03 \times 10^{47} \mathrm{~J}^{-1} \mathrm{~m}^{-3}$ 


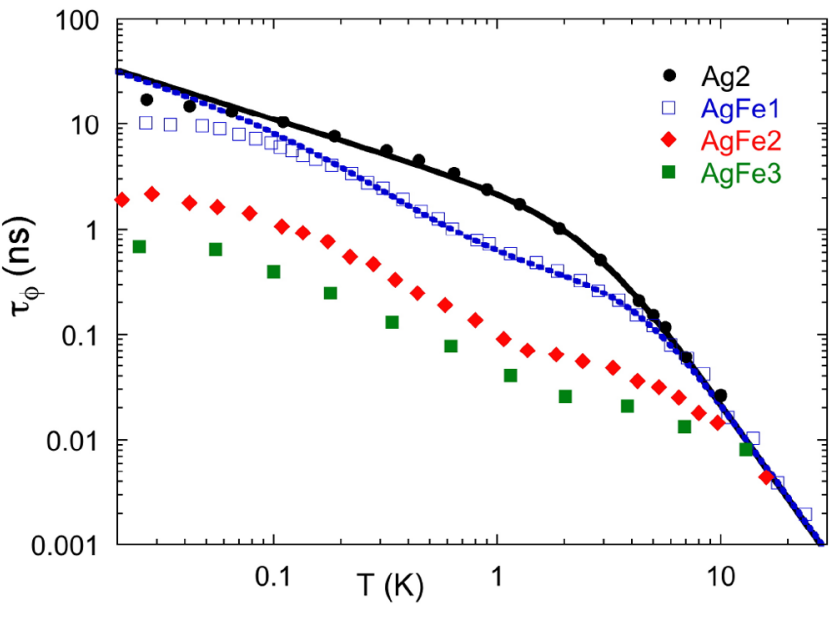

FIG. 2 (color online). Phase coherence time of Ag wires containing different amounts of Fe impurities. The solid line corresponds to the assumption that only electron-electron and electron-phonon interaction contribute to dephasing. The dotted line is a fit to the experimental data taking into account the magnetic contribution to the dephasing for $S=1 / 2$ impurities.

for the density of states [4]. We obtain $n_{s}^{\gamma}=1.3,13$, and $33 \mathrm{ppm}$ for sample $\mathrm{AgFe} 1, \mathrm{AgFe} 2$, and $\mathrm{AgFe} 3$, respectively. The Kondo temperature for all samples are on the order of $T_{K}^{\gamma}=4.3 \pm 0.2 \mathrm{~K}$.

To extract specifically the dephasing rate due to magnetic impurities $\gamma_{m}$, we subtract the experimentally measured electron-electron and electron-phonon contribution of the clean sample. We then plot our data scaled per magnetic impurity as a function of $T / T_{K}^{\gamma}$ as shown in Fig. 3. In addition, we also plot data of sample AuFe 2 of Ref. [11] having a different Kondo temperature. All data points fall on a universal curve. For comparison, we plot the NRG results for $S=1 / 2$ [9] as indicated by the arrow. We find remarkable agreement with this theory down to temperatures of $0.1 T_{K}^{\gamma}$. At lower temperatures, however, we observe deviations from this theory, as emphasized on the log-log plot in the inset of Fig. 3. The fact that we observe a universal curve for different impurity concentrations which depends only on $T / T_{K}$ is a strong indication that we are in the single impurity limit. On the other hand, it is clear that our data are not consistent with the Fermi liquid prediction of the spin $1 / 2$ impurity model where a $T^{2}$ [23] temperature dependence of the magnetic scattering time is expected at low temperature.

It is quite remarkable that the temperature dependence of $\gamma_{m}$ above $0.1 T_{K}$ is this well described by the $S=1 / 2$, single channel model. Fe is expected to be characterized by both an orbital degree of freedom and a much larger spin, $S=2$, coupling to electrons in up to five angular momentum channels. The relevant theoretical model depends on the position of the $\mathrm{Fe}$ within the $\mathrm{Ag}$ crystal structure, crystal field effects, and the strength of the spin-orbit coupling and is presently not known. An obvious candidate to explain the weak temperature dependence of $\gamma_{m}$ for low $T$ is to assume that the large spin of $\mathrm{Fe}$ is only partially

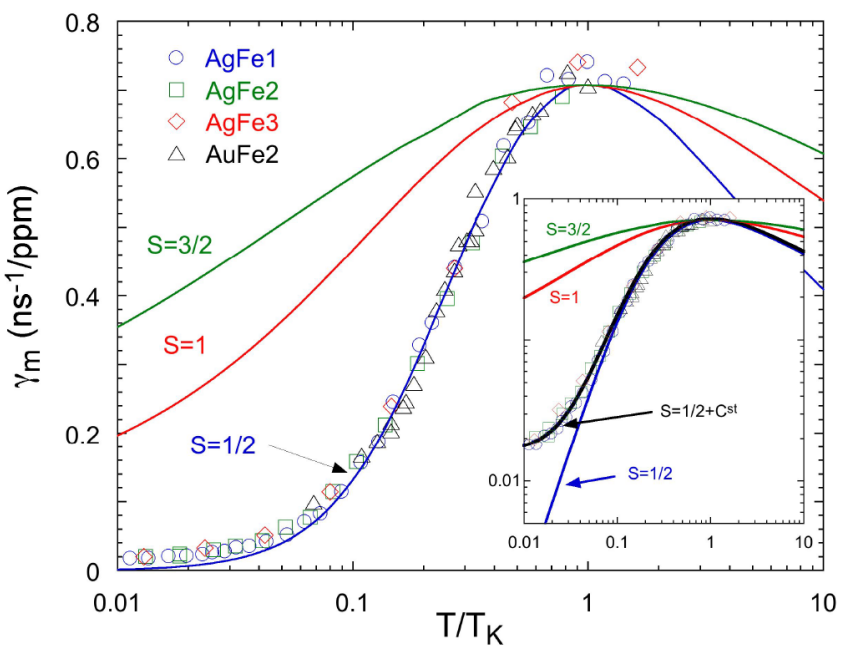

FIG. 3 (color online). Dephasing rate per magnetic impurity as a function of $\left(T / T_{K}^{\gamma}\right)$. The NRG results, keeping 1900 states for each NRG iteration and using the discretization parameter $\Lambda=$ 1.50 , for $S=1 / 2, S=1$, and $S=3 / 2$ have been scaled in temperature such that the maxima coincide with the experimental data. Inset: Same data on a log-log scale. The thick solid line corresponds to $S=1 / 2$ with a constant background added.

screened. This occurs when the effective number of channels $n_{c}$ is smaller than $2 S$ (for cubic symmetry $n_{c}=2$ or 3 is expected).

We have therefore computed $\gamma_{m}$ and the Kondo contribution to the resistance $R(T)$ for $1 / 2 \leq S \leq 5 / 2$ in the single channel, $n_{c}=1$, limit as shown in Figs. 1 and 3 for $S \leq 3 / 2$ [24]. Already for $S=1$, the temperature dependence of both, the resistivity as well as the dephasing rate is much slower than for the case of $S=1 / 2$. By screening a single channel, one can only reduce the size of the spin by $1 / 2$. The resulting spin of size $S-1 / 2$ remains, however, so strongly coupled to the electrons that $\gamma_{m}$ remains large even considerably below $T_{K}$. The corresponding very broad maximum around $T_{K}$ is clearly inconsistent with the experimental result already for $S=1$ as is shown in Fig. 3. While the resistivity is much less sensitive at low $T$, similar conclusions can be drawn by comparing our results for $\Delta \rho(T)$ to the NRG theory in Fig. 1.

The above analysis shows that the experimental data are indeed extremely close to a spin $1 / 2$ model and we can definitely rule out any underscreened scenario. Also an overscreened Kondo model with $n_{c}>2 S$ can not explain the experimental data as it would lead to an even larger dephasing rate at low $T$ [26]. Our data strongly indicate a perfect screening of almost all of the Fe impurities. Such a screening may, however, involve more than one channel and a spin larger than $1 / 2$ and also spin-orbit coupling. This may explain the differences between $T_{K}^{\rho}$ and $T_{K}^{\gamma}$ as well as $n_{S}^{\gamma}$ and $n_{s}$. Note that there is also a theoretical uncertainty in the prefactor of the dephasing rate and therefore in $n_{S}^{\gamma}$ in systems with strong variations of the Fermi velocity along the Fermi surface (as in $\mathrm{Ag}$ ) as discussed in Ref. [27]. 
What could then be the origin of this puzzling temperature dependence of $\gamma_{m}$ below $0.1 T_{K}$ ? One could argue that the relatively weak temperature dependence below $0.1 T_{K}$ originates from some other source, different from magnetic impurities. As $\gamma_{m}$ scales perfectly with the magnetic impurity concentration, we can definitely rule out any extrinsic effects. The curious temperature dependence of $\gamma_{m}$ has to originate from the magnetic impurities themselves or from the implantation process. A small fraction $(\leq 2 \%)$ of implanted Fe impurities may, for example, end up close to a lattice defect or in a grain boundary, "accidentially" giving rise to either a much lower Kondo temperature and/or a different crystal field or spin-orbit split state with much less screening and consequently an enhanced dephasing rate at the relevant low temperatures. One could also imagine that the ion implantation creates additionally dynamical defects, like two level systems (TLS) which can lead to a much slower decay of the dephasing rate at low temperatures [26]. Assuming a temperature independent background, arising from such slow TLS, as indicated by the thick solid line in the inset of Fig. 3, results in a very good agreement between the experimental data and the $S=1 / 2$ result. Let us mention, however, that electron spin resonance experiments, where $\mathrm{Cu}$ implantations have been performed into a $\mathrm{Cu}$ host, did not see any difference before and after implantation down to temperatures of $3 \mathrm{~K}$ [28].

In conclusion, we have measured the phase coherence time of $\mathrm{AgFe}$ quantum wires down to temperatures of $0.01 T_{K}$. We have shown that the magnetic contribution to the dephasing rate $\gamma_{m}$ per magnetic impurity is a universal function of $T / T_{K}$. Its temperature dependence is in remarkable agreement with recent NRG studies for $S=1 / 2$, down to temperatures of $0.1 T_{K}$. At lower temperatures, we observe deviations from this theory. The comparison of the experimental data with our NRG calculations for higher spins allows us to rule out a purely underscreened Kondo effect to explain the deviations at the lowest temperatures. Ion implantation of non magnetic impurities and further tests of scaling should allow us to shed light onto this puzzling temperature dependence of $\gamma_{m}$ in the very lowtemperature limit.

We acknowledge helpful discussions with P. Simon, G. Zaránd, L. Borda, L. Glazman, A. Zawadowski, S. Kettemann, J-L. Tholence, O. Laborde, K. Matho, A. Altland, P.H. Dederichs, A. Liebsch, H. Bouchiat, M. Lavagna, and D. Feinberg. We are indebted to the Quantronics group for the silver evaporation. This work has been supported by the European Commission FP6 NMP-3 Project 505457-1 Ultra-1D and ANR-PNANO QuSpin and by SFB 608 and TR12 of the DFG.

Note added. - We recently became aware of a related work on the same topic [21].
[1] A.C. Hewson, The Kondo Problem to Heavy Fermions (Cambridge Studies in Magnetism, Cambridge, UK, 1997).

[2] J. Kondo, Prog. Theor. Phys. 32, 37 (1964).

[3] P. Nozières, J. Low Temp. Phys. 17, 31 (1974).

[4] F. Pierre et al., Phys. Rev. B 68, 085413 (2003).

[5] P. Mohanty and R. A. Webb, Phys. Rev. Lett. 84, 4481 (2000); and , 91, 066604 (2003).

[6] F. Pierre and N. O. Birge, Phys. Rev. Lett. 89, 206804 (2002).

[7] F. Schopfer, C. Bäuerle, W. Rabaud, and L. Saminadayar, Phys. Rev. Lett. 90, 056801 (2003).

[8] R. P. Peters, G. Bergmann, and R. M. Mueller, Phys. Rev. Lett. 58, 1964 (1987); C. Van Haesendonck, J. Vranken, and Y. Bruynseraede, Phys. Rev. Lett. 58, 1968 (1987).

[9] T. Micklitz, A. Altland, T. A. Costi, and A. Rosch, Phys. Rev. Lett. 96, 226601 (2006).

[10] G. Zaránd, L. Borda, J. v. Delft, and N. Andrei, Phys. Rev. Lett. 93, 107204 (2004).

[11] C. Bäuerle, F. Mallet, F. Schopfer, D. Mailly, G. Eska, and L. Saminadayar, Phys. Rev. Lett. 95, 266805 (2005).

[12] C. Rizutto, Rep. Prog. Phys. 37, 147 (1974).

[13] The SRIM simulations indicate that $22 \%$ of the ions penetrate into the substrate. This correction is taken into account for the determination of $n_{s}$ (Table I).

[14] B. L. Altshuler, A. G. Aronov, and D.E. Khmelnitzky, J. Phys. C 15, 7367 (1982).

[15] E. Akkermans and G. Montambaux, Mesoscopic Physics of Electrons and Photons (Cambridge University Press, Cambridge, UK, 2007).

[16] T. A. Costi, A. C. Hewson, and V. Zlatic, J. Phys. Condens. Matter 6, 2519 (1994).

[17] T. A. Costi, Phys. Rev. Lett. 85, 1504 (2000).

[18] S. Hikami, A. I. Larkin, and Y. Nagaoka, Prog. Theor. Phys. 63, 707 (1980).

[19] Note that the prefactor of the magentic part may differ by a factor of $2[8,11]$ depending on whether the spin relaxes faster or slower than $\tau_{m}[20,21]$.

[20] M. G. Vavilov, L. I. Glazman, and A. I. Larkin, Phys. Rev. B 68, 075119 (2003).

[21] G. M. Alzoubi and N. O. Birge, Phys. Rev. Lett. 97, 226803 (2006).

[22] This number is about 2 times larger than $a_{\text {theo }}=$ $0.22 \mathrm{~ns}^{-1} \mathrm{~K}^{-2 / 3}$.

[23] At very low $T$, one expects $1 / \tau_{m} \sim T^{3 / 2}$ due to weak localization corrections to $1 / \tau_{m}[9]$.

[24] Similar calculations for the frequency-dependence of the inelastic cross section at $T=0$ can be found in Ref. [25], analytical results for $\tau_{m}$ at low and high $T$ are discussed in [20]).

[25] W. Koller, A. C. Hewson, and D. Meyer, Phys. Rev. B 72, 045117 (2005).

[26] A. Zawadowski, J.v. Delft, and D. C. Ralph, Phys. Rev. Lett. 83, 2632 (1999).

[27] T. Micklitz, T. A. Costi, and A. Rosch, cond-mat/0610304.

[28] P. Monod, H. Hurdequint, A Janossy, J. Obert, and J. Chaument, Phys. Rev. Lett. 29, 1327 (1972). 